\title{
Increases in exposure calls related to selected cleaners and disinfectants at the onset of the COVID-19 pandemic: data from Canadian poison centres
}

\author{
Abdool Yasseen III, PhD (1); Deborah Weiss, PhD (1); Sandy Remer, MD (1,2); Nina Dobbin, MSc (1,3); \\ Morgan MacNeill, MSc (1,4); Bojana Bogeljic, MA (1); Dennis Leong, BSc(Pharm) (3); Victoria Wan, MSc (3); \\ Laurie Mosher, RN (4); Guillaume Bélair, MSc (5); Margaret Thompson, MD (2); Brooke Button, BScN (6); \\ James Hardy, BSc (7); Shahid Perwaiz, PhD (8); Alysyn Smith, RN (8); Richard Wootton, BA (1)
}

Published online September 23, 2020

Tweet this article

\begin{abstract}
Little is known about the use or misuse of cleaning products during the COVID-19 pandemic. We compiled data from January to June in 2019 and 2020 from Canadian poison centres, and report on calls regarding selected cleaning products and present year-overyear percentage change. There were 3408 (42\%) calls related to bleaches; 2015 (25\%) to hand sanitizers; 1667 (21\%) to disinfectants; 949 (12\%) to chlorine gas; and 148 $(2 \%)$ to chloramine gas. An increase in calls occurred in conjunction with the onset of COVID-19, with the largest increase occurring in March. Timely access to Canadian poison centre data facilitated early communication of safety messaging for dissemination to the public.
\end{abstract}

\section{Introduction}

The first Canadian case of novel coronavirus disease 2019 (COVID-19), caused by infection with SARS-CoV-2, was recorded on 15 January, 2020, and at the time of writing in August 2020, 117792 COVID-19 laboratory-confirmed cases had been reported, including 8958 deaths. ${ }^{1,2}$ Through February and March 2020, messaging from Canadian public health officials increasingly focussed on strategies to reduce the spread of SARS-CoV-2, including physical distancing, proper cough etiquette, hand-washing, use of alcohol-based hand sanitizers and cleaning and disinfecting of high-touch surfaces. ${ }^{3}$ On 11 March, 2020, the World Health Organization formally declared the COVID-19 epidemic to be a global pandemic, ${ }^{4}$ and in the following days, federal, provincial and territorial and municipal authorities across Canada implemented stringent physical distancing measures, including travel restrictions, the temporary closures of businesses and schools, and the cancellation of nonurgent medical appointments and procedures. Daily messages from public health officials at all levels of government reinforced the urgency of taking steps to limit the spread of SARS-CoV-2.

With an increased focus on cleaning and disinfecting comes the possibility of increased exposure to chemicals in cleaning products; more specifically, to the fumes and by-products created by the inappropriate combination of these products. ${ }^{5,6}$ In a report from the US Centers for Disease Control and Prevention, the

\section{Highlights}

- The Canadian Surveillance System for Poison Information (CSSPI) led by Health Canada is a developing network of poison centres, health authorities and regulatory agencies that facilitates early detection of poisoning incidents and alerting at the national level to inform harm reduction interventions.

- In response to the COVID-19 pandemic, concerns were raised over the potential for misuse of cleaning products and disinfectants; the CSSPI network monitored and assessed these concerns.

- An overall increase in calls about select cleaning products and disinfectants occurred concurrently with the pandemic, with percentage increases for selected products as high as $400 \%$ compared to the same period in the previous year.

authors reported an increase in calls to poison centres related to exposures to cleaners and disinfectants for the first three months of 2020, compared to the same period in 2019. ${ }^{5}$ While increases in exposures were reported for all age groups, exposures in young children

\section{Author references:}

1. Chemical Emergency Management and Toxicovigilance Division, Health Canada, Ottawa, Ontario, Canada

2. Ontario Poison Centre, Toronto, Ontario, Canada

3. British Columbia Centre for Disease Control, Drug and Poison Information Centre, Vancouver, British Columbia, Canada

4. Izaak Walton Killam Poison Centre, Halifax, Nova Scotia, Canada

5. Centre antipoison du Québec, Québec City, Quebec, Canada

6. Poison and Drug Information Service, Calgary, Alberta, Canada

7. Consumer and Hazardous Products Safety Directorate, Health Canada, Ottawa, Ontario, Canada

8. Health Products Food Branch, Health Canada, Ottawa, Ontario, Canada

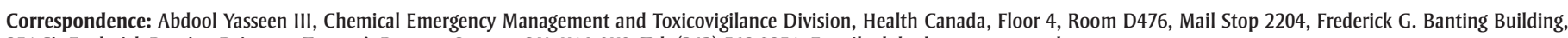
251 Sir Frederick Banting Driveway, Tunney’s Pasture, Ottawa, ON K1A 0K9; Tel: (343) 542-8251; E-mail: abdool.yasseen@canada.ca 
consistently represented a large proportion of the calls. Poison centres in France raised similar issues, hypothesizing that the increased presence of young children in the home, combined with changes in cleaning behaviour, might be leading to increases in poisonings. ${ }^{6}$ In Canada, little is known about the reporting of these products, especially during pandemic periods; in this report we present data from the five poison centres across Canada.

\section{Methods}

We report on de-identified data obtained from the five Canadian poison centres through a data request process, and compiled by the Canadian Surveillance System for Poison Information (CSSPI) program led by Health Canada. These data consist of information collected on exposure calls received from January to June 2019, and from January to June 2020, for which exposures were identified using the American Association of Poison Control Centers (AAPCC) codes for hand sanitizers: 200613, 200614, 200615 and 200616; disinfectants: 39282, 40280, 42281 and 77286; bleaches: 42280, 62280, 77280 and 77282; chlorine gas: 116400 and 116401; and chloramine gas: 77403 . Exposure calls were presented as counts overall and stratified by age of the exposed individual (i.e. $\leq 19$ years, and adults $\geq 20$ years) and assessed monthly for all five poison centres between January and June for the years 2019 and 2020, and weekly for four of the five poison centres between March and April 2020. We calculated percentage change in year-over-year estimates as follows:

$\% \Delta_{\text {year-to-year }}=\frac{\left(\text { Month }_{2020}-\text { Month }_{2019}\right)}{\text { Month }_{2019}} * 100 \%$

Note that for one poison centre, data were unavailable prior to 6 February 2019, meaning that information presented at the national level is partially missing for the specified time period. We contacted the Health Canada Research Ethics Board (REB) to discuss the CSSPI initiative, and determined that a REB review was not required, given the mandatory suppression of small cell sizes that ensures minimal risk of re-identification.

\section{Results}

There were a total of 8187 calls reporting exposures between January and June for
2019 and 2020. Of these, 3408 (42\%) calls were related to bleaches; 2015 (25\%) to hand sanitizers; 1667 (21\%) to disinfectants; 949 (12\%) to chlorine gas; and 148 (2\%) to chloramine gas. We observed a $35 \%$ rise in the total number of exposures related to these cleaning products in January 2020 when compared to January 2019, but no apparent difference for February. However, there was significant heterogeneity among the various types of cleaning products in February 2020, with hand sanitizers and disinfectants each showing an increase of approximately $40 \%$, and bleaches, chlorine gas and chloramine gas showing decreases of $3 \%, 9 \%$ and $12 \%$, respectively, compared to February 2019 (Figure 1). We observed greater increases for the months of March to June, particularly among disinfectants and chloramine gas exposures. Weekly estimates show that the number of calls peaked during the week of 22 March 2020, predominantly among those aged older than 19 years (Figure 2) and there was a general decrease in the percentage change after April 2020, with the exception of hand sanitizers and chloramine gas.

\section{Discussion}

Using data from Canadian poison centres, we observed an increase in exposure calls concerning selected cleaners and disinfectants in March 2020 compared to March 2019. Weekly data collected from March through May 2020 suggest the increase peaked in the third week of March, shortly after the WHO declared the global pandemic. These results are consistent with reports from the US, and given the timing, may indicate increased exposures to these products associated with the onset of the COVID-19 global pandemic. ${ }^{5}$ The reason(s) for this increase are unclear, but may be related to an increased use of cleaning products in an attempt to mitigate risk of infection from COVID-19. Additionally, limited availability of certain cleaners could lead to inappropriate use of or mixing of other products, as well as misuse of products for personal hygiene or decontamination. ${ }^{6}$ With the closures of schools and daycares, young children were at home, which might be expected to create greater opportunity for exposure. However, our results do not suggest that there was a large increase among those aged 19 years or younger. Percentage changes in January and February are possibly related to pre-pandemic concerns, but are more likely incidental as the magnitude of these differences is not substantial.

Our results indicate an opportunity for proactive messaging to effectively communicate the potential risks related to cleaners and disinfectants during the COVID-19 pandemic. In reaction to early surveillance data, public health officials and regulatory agencies advocated for the safe use of these products, and stressed the importance of timely access to poison centre data at the national level. Following initial observations in March 2020, we held discussions with the five poison centres and the Canadian federal government programs responsible for improving the safety of the selected cleaning products. This preliminary meeting was used as a starting point to generate messaging (both for social media and for online dissemination) aimed at reinforcing the safe use of those cleaners and disinfectants. Between March and June 2020, Health Canada deployed a number of communication tactics in order to provide information to Canadians on the safe use of household cleaners, disinfectants and hand sanitizers. This included concurrent social media campaigns broadcast through various departmental channels on Twitter, Facebook and LinkedIn. Social media content encouraged the cleaning of hands and surfaces, and provided information on how to do so safely. Social media content also featured information on the risks of making hand sanitizers at home and mixing cleaning products together, and on using bleach safely and the importance of keeping these types of products away from children. In addition to social media, Health Canada developed a web portal (https:// www.canada.ca/en/health-canada/services /drugs-health-products/disinfectants /covid-19.html) dedicated to hand sanitizers and disinfectants. This website is updated regularly and includes information on actions taken by the Government of Canada to increase the supply of these products, as well as how to use them safely. Furthermore, Health Canada has issued a variety of public advisories and news releases on the importance of cleaning, disinfecting and sanitizing hands and surfaces safely.

\section{Strengths and limitations}

A major strength of this work is that it combines regional information and expertise from Canadian poison centres to report on national statistics across Canada. 
FIGURE 1

Number of calls made to Canadian poison centres regarding selected cleaning products and disinfectants in 2019 and 2020 (January to June), with year-over-year percentage changes
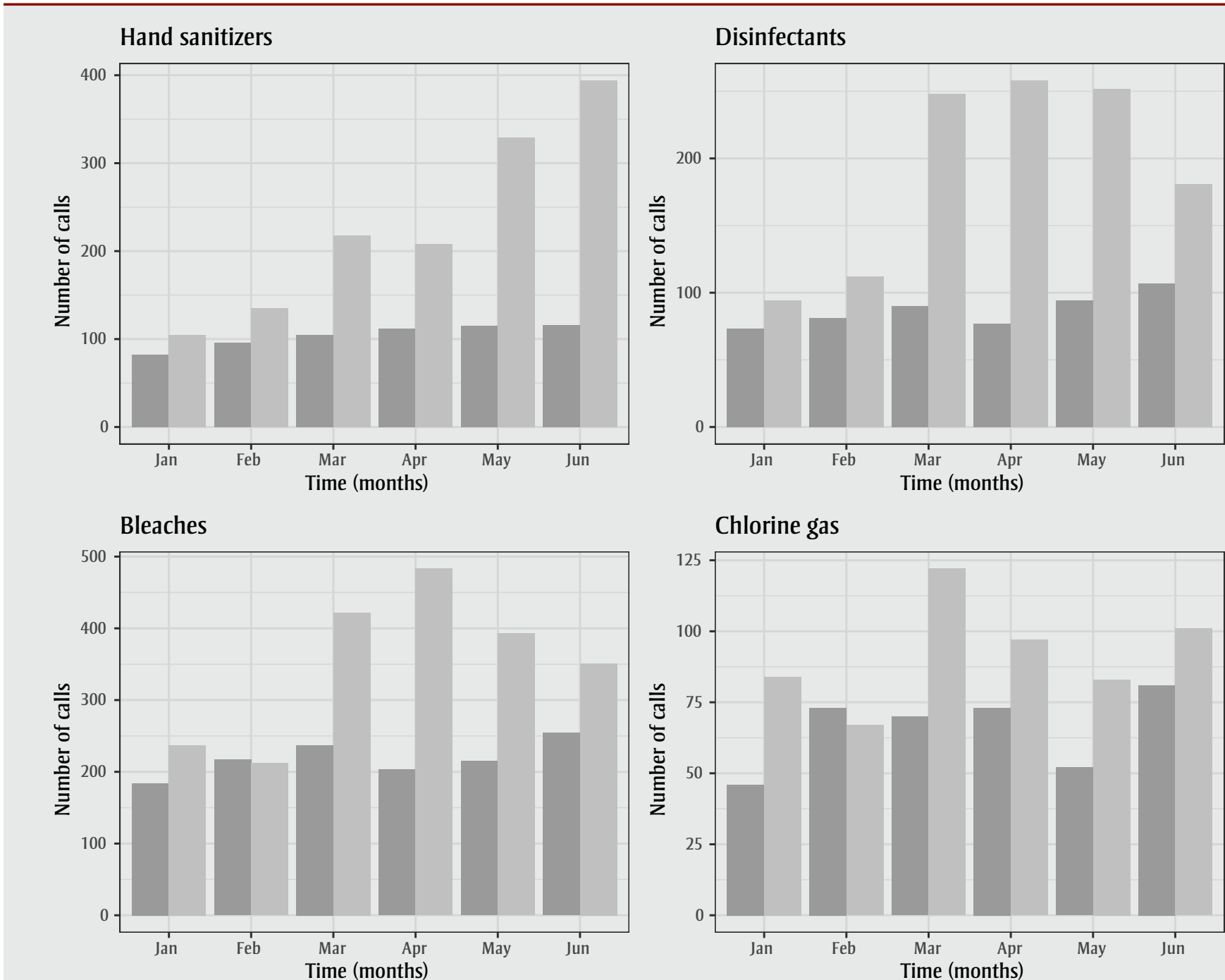

\section{Chlorine gas}

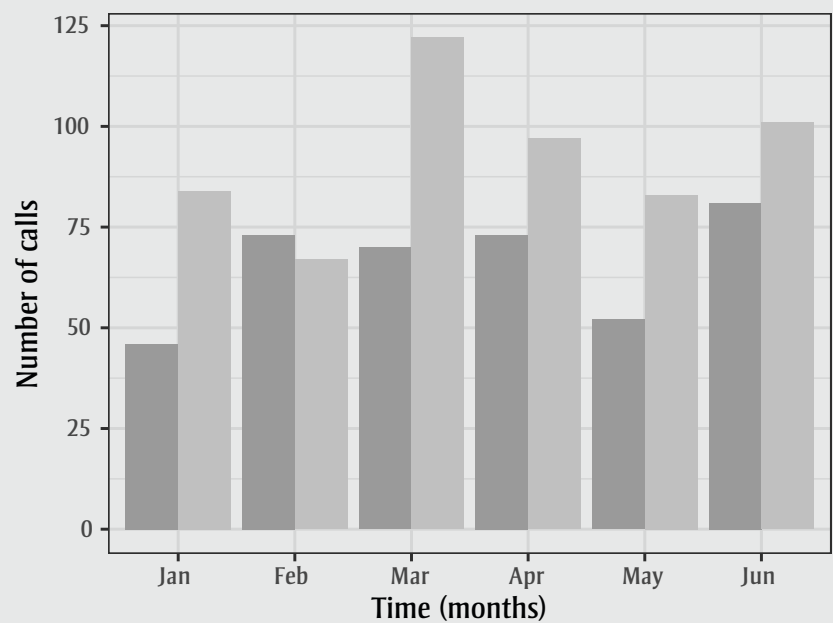

Chloramine gas

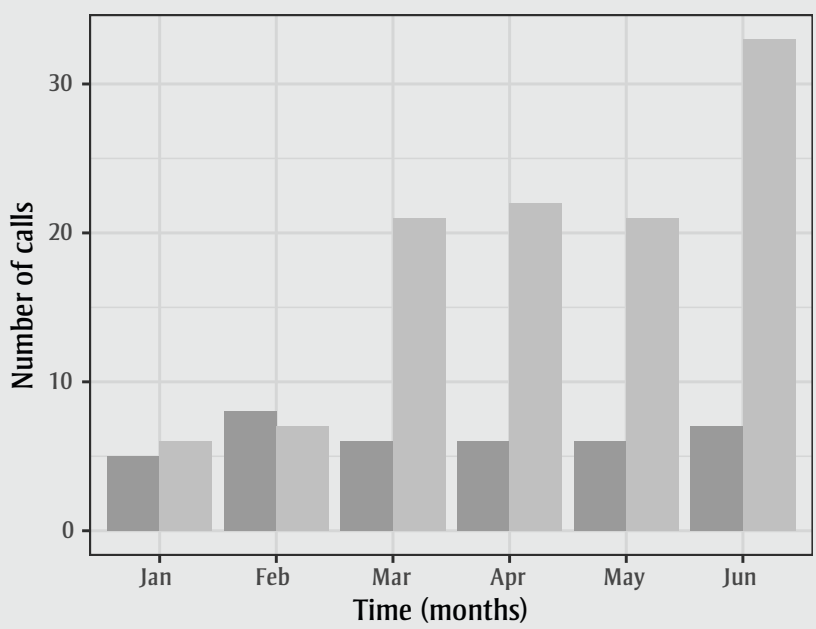

Percentage change (2020 vs. 2019)

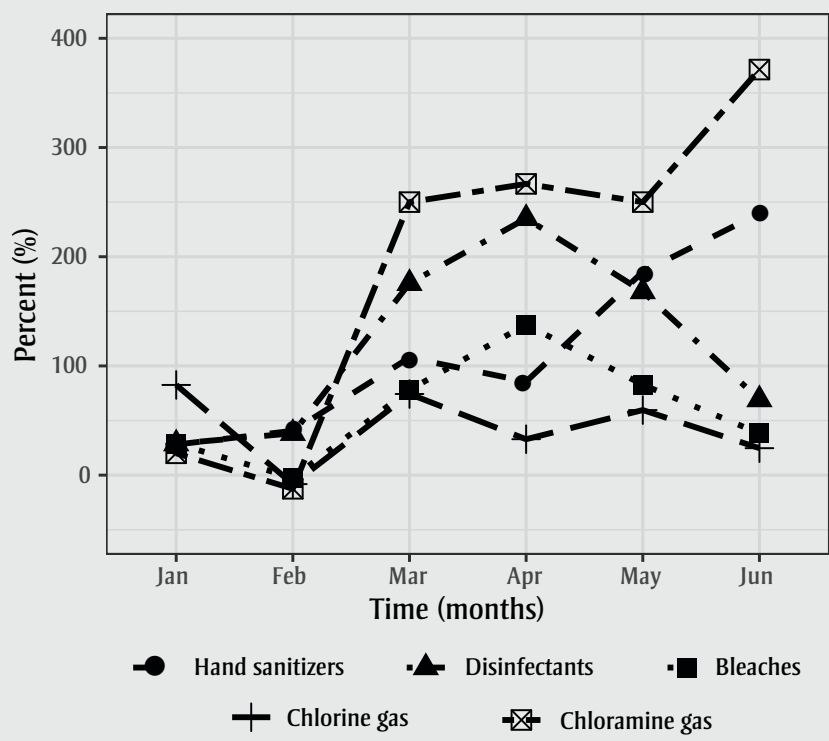


FIGURE 2

Weekly exposure call counts for all exposure calls regarding cleaning products in 2020, stratified by age group and cleaning products, for four of the five Canadian poison centres ${ }^{\mathrm{a}}$

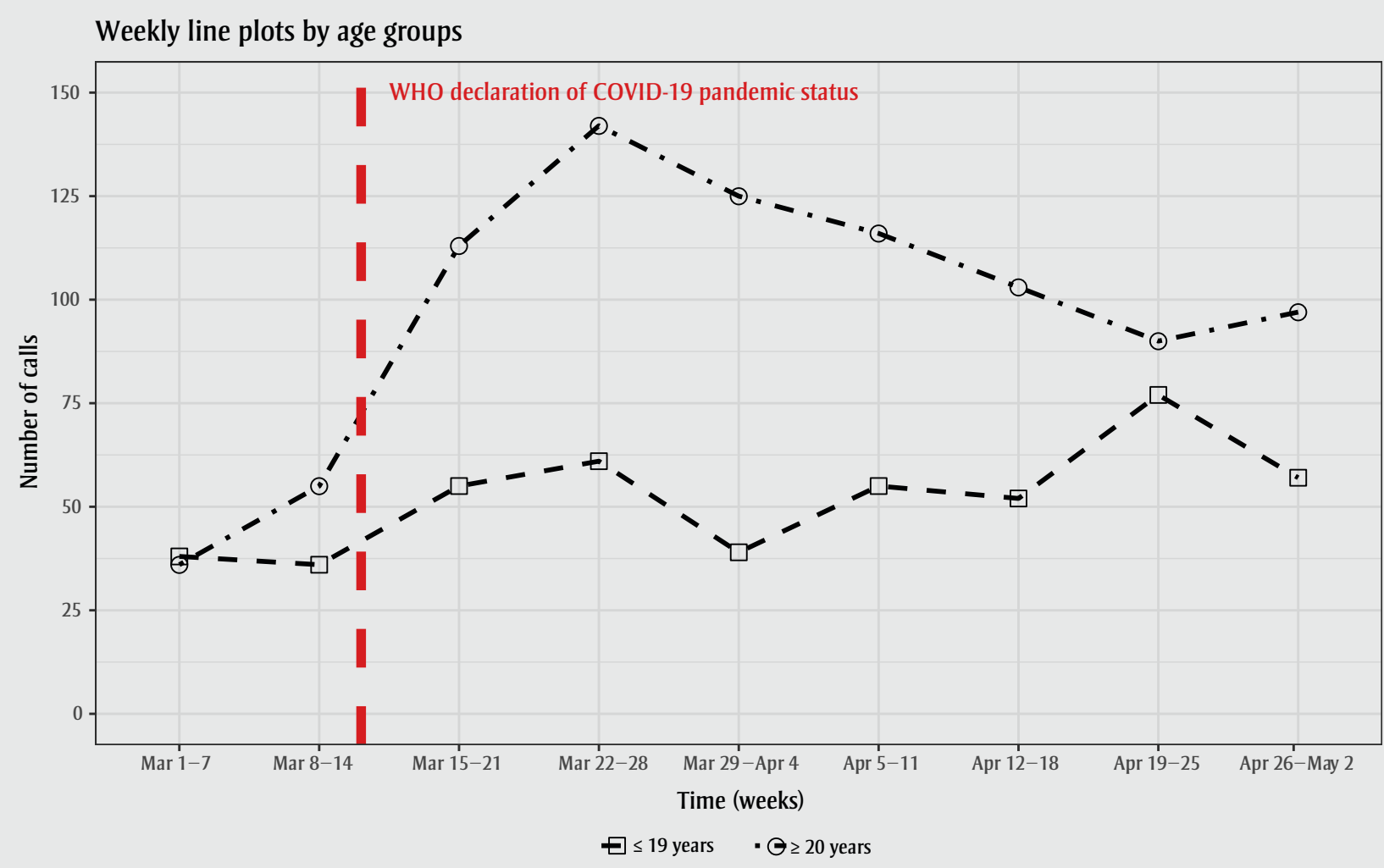

Weekly line plots by cleaning products and disinfectants

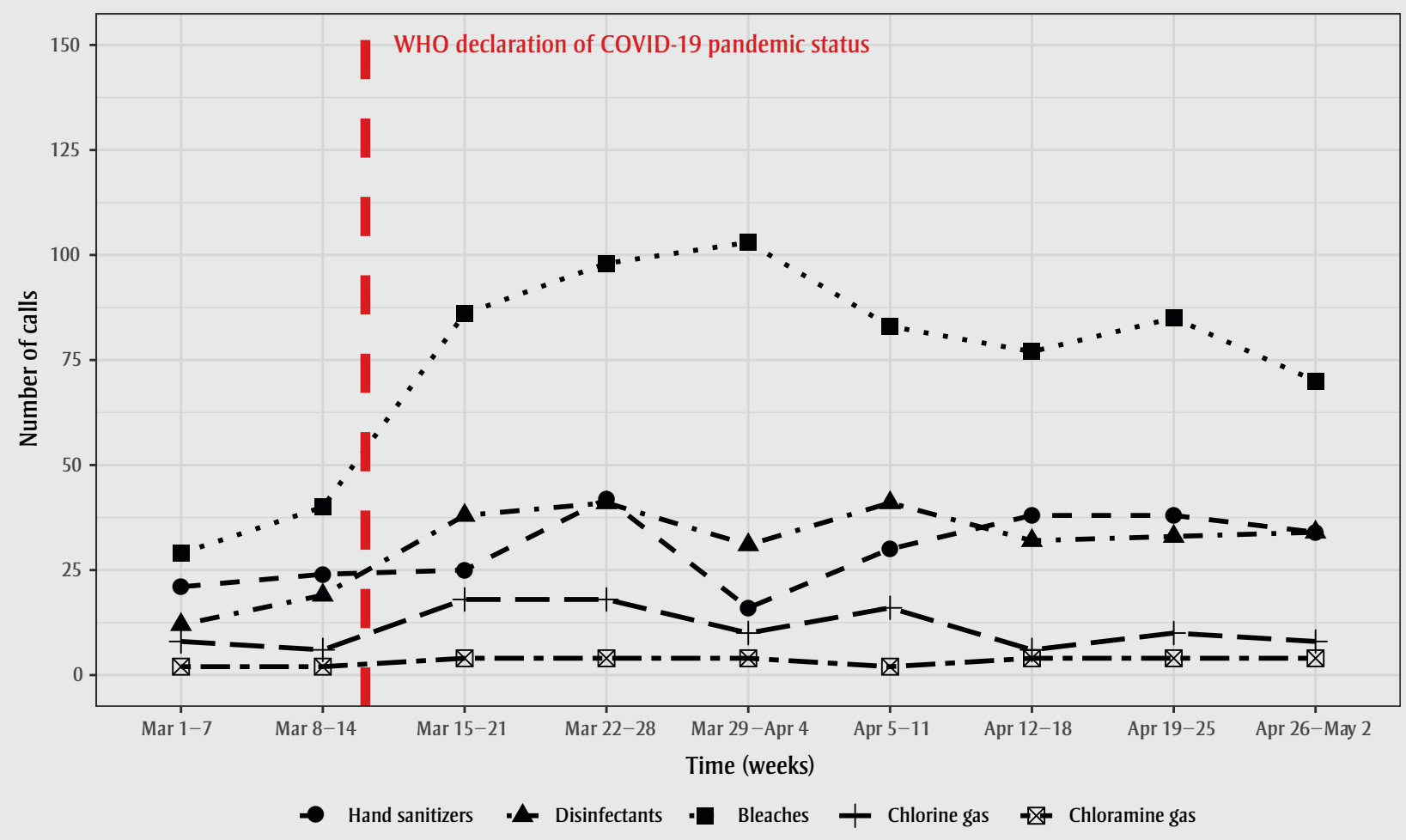

a One poison centre was unable to contribute weekly data and was excluded from this analysis. 
We disseminated preliminary versions of this report through the CSSPI network, which includes clinical specialists, public health officials and personnel from regulatory agencies, in order to collectively develop guidelines and enable knowledge translation and the exchange of messaging on the appropriate use of these products. Additionally, this report makes use of poison centre information that is unavailable from other health administrative data sources and remains a relatively untapped resource for understanding the epidemiology of environmental exposures in Canada.

However, as with any secondarily collected data, there are potential biases that may affect interpretation of the observed results. Our analyses are based on calls made to Canadian poison centres, and likely only represent a small portion of total exposures, as some will self-manage at home while others will directly seek inperson medical attention. Furthermore, Specialists in Poison Information at the poison centres rely on self-reported responses to treat or manage adverse exposures. Accordingly, the information documented in their case management systems is subject to the ability of the caller to accurately describe the exposure. Most, but not all, regions in Canada have access to a poison centre; therefore, the data are not fully represented nationally. However, the information presented in this report represents our best estimates at the time of publication. Additionally, at the time of publication, historical data on cleaning products and disinfectants prior to 2019 was unavailable and precluded further statistical analysis. While efforts to improve poison centre data quality and comparability are ongoing, inconsistencies in coding between and within poison centres do exist. Lastly, these results show an association between increases in exposure calls to Canadian poison centres related to cleaners and disinfectants and the COVID-19 pandemic in Canada; however, causality should not be inferred as this relationship is not well understood and there may yet be unknown influential drivers.

\section{Conclusion}

An increase in calls to poison centres regarding selected cleaners and disinfectants occurred in conjunction with the onset of the COVID-19 pandemic in Canada. This work highlights the importance of timely access to poison centre data at the national level. Calls to poison centres represent a relatively untapped resource for understanding Canadian exposures to potentially hazardous chemical substances, as this information is not readily available via health administrative data sources.

\section{Acknowledgments}

This work would not have been possible without the work and support of the specialists in poison information at the five Canadian poison centres. Their diligent efforts to provide expert advice to the public and clinical practitioners alike facilitated the collection and documentation of the information required to conduct these surveillance activities.

\section{Conflicts of interest}

The authors declare no real or perceived conflict(s) of interest.

\section{Authors' contributions and statement}

AY, DW, SR, ND, MM and RW conceived and planned the study and developed the protocol. AY, DW, SR, ND, and MM developed the analysis plan and analyzed the data. AY and DW drafted the initial article, distributed this draft to SR, ND, MM, and RW for initial review, then to all other listed authors for quality checks, appraisal of results, and review of text. All authors helped critically revise the article throughout the development and review process.

The content and views expressed in this article are those of the authors and do not necessarily reflect those of the Government of Canada.

\section{References}

1. Government of Canada. Coronavirus disease 2019 (COVID-19): epidemiology update [Internet]. Ottawa (ON): Government of Canada; [modified 2020 Aug 24; cited 2020 Aug 12]. Available from: https://health-infobase.canada .ca/covid-19/epidemiological-summary -covid-19-cases.html

2. Government of Canada. Coronavirus disease (COVID-19): outbreak update [Internet]. Ottawa (ON): Government of Canada; [modified 2020 Aug 04; cited 2020 Aug 12]. Available from: https://www.canada.ca/en/public -health/services/diseases/2019-novel -coronavirus-infection.html?topic $=$ tilelink
3. Government of Canada. Coronavirus disease (COVID-19): prevention and risks [Internet]. Ottawa (ON): Government of Canada; [modified 2020 Aug 19; cited 2020 Aug 12]. Available from: https://www.canada.ca/en /public-health/services/diseases/2019 -novel-coronavirus-infection/prevention -risks.html

4. World Health Organization (WHO). WHO Director-General's opening remarks at the media briefing on COVID-19 - 11 March 2020 [Internet]. Geneva (CH): WHO; 2020 Mar 11 [cited 2020 Aug 12]. Available from: https://www.who.int/dg/speeches /detail/who-director-general-s -opening-remarks-at-the-media -briefing-on-covid-19---11-march-2020

5. Chang A, Schnall AH, Law R, et al. Cleaning and disinfectant chemical exposures and temporal associations with COVID-19-National Poison Data System, United States, January 1, 2020-March 31, 2020. MMWR Morb Mortal Wkly Rep. 2020;69(16):496-8.

6. Le Roux G, Sinno-Tellier S, French Poison Control Centre members, Descatha A. COVID-19: home poisoning throughout the containment period. Lancet Public Health. 2020: 5(6):e314. Epub 2020 Apr 25. Erratum in: Lancet Public Health. 2020 May 4:5(6):e315. 